\title{
Using Date Seed Powder Nanoparticles and Infusion as a Sustainable Source of Nutraceuticals
}

\author{
Amany Alsayed Salama ${ }^{1, *}$, Naglaa Mohamed Ismael', Mervat Mostafa Megeed ${ }^{2}$ \\ ${ }^{1}$ Department of Nutrition, Faculty of Allied Medical Sciences, Pharos University in Alexandria, Alexandria, Egypt \\ ${ }^{2}$ Department of Medical Devices, Faculty of Allied Medical Sciences, Pharos University in Alexandria, Alexandria, Egypt

\section{Email address:} \\ amany.salama@pua.edu.eg (A. A. Salama),naglaa.esmail@pua.edu.eg (N. M. Ismael), mervat.mostafa@pua.edu.eg (M. M. Megeed) \\ ${ }^{*}$ Corresponding author
}

\section{To cite this article:}

Amany Alsayed Salama, Naglaa Mohamed Ismael, Mervat Mostafa Megeed. Using Date Seed Powder Nanoparticles and Infusion as a Sustainable Source of Nutraceuticals. Journal of Food and Nutrition Sciences. Vol. 7, No. 3, 2019, pp. 39-48.

doi: $10.11648 /$ j.jfns.20190703.11

Received: August 1, 2019; Accepted: August 22, 2019; Published: September 5, 2019

\begin{abstract}
Egypt is the world's largest date producing country. Different functional foods pertaining to date seeds were attempted before. Phenolic phytochemicals are not well absorbed in gastrointestinal tract, which results in lower bioactivity. Nanotechnology helps to resolve poor oral bioavailability. Aim was to evaluate the in-vivo anti-inflammatory properties of date seed powder (DSP) nanoparticles (NPs) and infusion in rats fed a high fat diet (HFD). Twenty five Wistar rats were distributed among five groups; I (control), II (on HFD), III (on HFD and DSPNP1 1:5), IV (on HFD and DSPNP2 2:3), and V (on HFD and DSP infusion $1 \mathrm{~g} / \mathrm{kg} /$ day). Blood samples were tested for C-reactive protein (CRP), Serum amyloid-A (SAA), total cholesterol, HDL, albumin, creatinine, and some primary biochemical parameters. HFD induced significant elevations in cholesterol and inflammatory markers as compared to the controls; while, HDL, ALT, AST, creatinine and albumin showed no change in all rats. In all DSP treatment groups, total cholesterol levels were reduced significantly. Both DSP NPs and infusion caused significant decrease in CRP and SAA levels. DSP NPs exhibited the same anti-inflammatory effects as their free counterparts. Using polyvinyl alcohol NPs of DSP in a wt/wt ratio of 2 PVA: 3 DSP; resulted in an acceptable particle size and physicochemical properties.
\end{abstract}

Keywords: Date Seeds, Nanotechnology, Phenolic Phytochemicals, Nutraceuticals \& Inflammatory Markers

\section{Introduction}

\subsection{Achieving Sustainable Health Through Healthy Foods with Lower Food Wastes}

The concept of 'Sustainable Health' was recently proposed by Del Castillo et al, as a healthy and active ageing avoiding the risk of diseases. To accomplish this, healthy foods and especially functional foods are required. Sustainable health can be achieved without depleting natural assets or causing serious ecological harm by providing high quality medical attention and improving population health. The protection and improvement of health now and for future generations through various approaches, such as healthy eating that could be focused on functional food items, can also achieve a sustainable health. [1] In addition, personalized food processing; reformulation as an example, can lead to more resource effective production of food with lower food wastes. [2] The growing trend in waste production in the agricultural food industry is a major global issue in view of its storage, disposal and environmental and potential health risks. Nevertheless, residues or by-products are an innovative way towards industrial growth and waste management to recover the added-value substances using inexpensive raw materials. [3]

\subsection{Nutraceuticals}

Nutraceuticals are generally food or parts of food which play an important role in the modification and retention of a proper, healthy individual's physiological function. Global population and health trends are key factors for the development of the nutraceutical industry. Dietary fiber, prebiotics, probiotics, poly-unsaturated fatty acids and various kinds of herbal or natural foods can be classified as 
nutraceuticals. These nutraceuticals assist to combat some of the millennium main health issues such as obesity, cardiovascular disease, diabetes, cholesterol, and cancer. All in all, "nutraceuticals," in the food-related sciences, led to the new era in medicine and well-being. [4]

\subsection{Date Seeds Added Value}

The fruit of Phoenix dactylifera (L.) is regarded as one of earth's oldest and nutrient-rich fruits. Its continuously increasing production and consumption yield accounts for approximately $10-15 \%$ of the total fruit manufacturing with concerns for disposal. Several scientists have examined the chemical makeup and profile of phytochemicals that have demonstrated in vitro bioactivities. Different functional foods and drinks pertaining to date seeds were also attempted. Their waste by-products use has a key role to play in the sustainable use of agricultural products. Many studies investigated date seed powder in multiple food recipes, as a cheap and nutritionally rich source of fibers and antioxidants. Though, a quantitative assessment of process escalation remains essential in profitable food marketing. [5] Egypt is the largest date producing country in the world since 1974, and produces yearly about 1.5 million tonnes that makes $20 \%$ of world's dates' production. [6]

Significant quantities of useful dietary components such as oleic acid, dietary fibers and polyphenols are found in date pits. The decreased incidence of heart disease as well as the enhanced general health and the use of these compounds have been correlated. Date seeds were lately utilized without sensory deterioration for the manufacturing of staple foods such as bread and hot drinks. Used as a raw material for functional food an undesirable residue of the date industry has excellent financial capacity and is anticipated in the next few years to improve. [7]

Studies show that consumption of date seeds may boost the body's antioxidant status as their flavones; polyphenol, unsaturated fatty acid, vitamin $\mathrm{C}$ and vitamin $\mathrm{E}$, and fiber content are proving useful as anti-atherogenic. Through antiinflammatory and anti-bacterial effects; antioxidants stop free radicals oxidation and decrease oxidized LDL. [8]

For their significant health enhancement; dietary phytochemicals are extensively researched medicine, nutrition, chemistry, and biology. Moreover, many laboratory and animal research demonstrate that a number of these compounds contribute to chronic illnesses like cancer and cardiovascular disease (CVD) prevention or control. [9]

\subsection{Obesity, Low Grade Inflammation and Oxidative Stress as Induced by a High-fat Diet}

Globesity; the global obesity epidemic pertaining to nutritional affluence, due to the use of a high-fat diet (HFD), is increasingly seen as a major modifiable risk factor for lifestyle related illnesses like diabetes, CVD and some cancers. The mechanisms underlying the impacts of HFDs on human health remain unclear. The development of obesity may be stimulated by dietary fat composition as a result of the precise function of some fatty acids with varying metabolic behavior that may change fat oxidation and deposition rates, leading to change in body weight or composition. [10]

Chronic inflammation is associated with many human conditions. Epidemiological and experimental studies have shown that modification by natural phytochemicals of inflammatory reaction plays a significant role in preventing, and treating several chronic inflammatory diseases. Flavonoids are a class of natural substances that have strong biological actions commonly found in vegetables, fruit and culinary plants. Flavonoid dietary intakes are recommended to avoid and reduce the risk of chronic diseases. [11]

\subsection{Anti-inflammatory Activity of Phenolic Phytochemicals}

A number of mechanisms have been suggested to elucidate the anti-inflammatory activity of flavonoids; e.g., by modulating inflammatory signals, reducing inflammatory molecules output, reducing induction and inflammatory cell proliferation, regulating cell function and antioxidant properties. With regard to safety issues, capacity and antiinflammatory impacts of flavonoids, it is possible that in chronic inflammatory circumstances they play a potentially preventive and therapeutic function. [12]

Multiple nutrition and pharmaceutical advantages are demonstrated by phenolic phytochemicals; for example, in the prevention of chronic diseases, delaying aging process, and reducing cancer and other diseases. Phenolic phytochemicals are not well absorbed in gastrointestinal tract, which results in lower bioactivity because of poor water solubility, reducing settlements with epithelial cells and epithelium transport in the small bowel. [13] Most importantly, phenolic phytochemicals cannot be recognized by a precise receptor on epithelial cells, and are transported by passive distribution. Thus it is well recognized that bioavailability can vary among different phenolic acids, and the dietary load of a specific substance does not inevitably lead to finest bioavailability profile. [14]

\subsection{Overcoming the Poor Oral Bioavailability of Phenolic Phytochemicals by Nanotechnology}

The development of nanosized carriers is a valuable strategy to resolving the poor oral bioavailability of ingredients. Nanoparticles have been extensively studied as delivery systems in the field of pharmaceutical science because their unique physicochemical properties provide them with cellular uptake features compared to bulk materials. [15] Particles are characterized by their diameter; nanoparticles have diameters from 1 to $100 \mathrm{~nm}$. Nevertheless, pharmaceutical science also classifies particles less than $1000 \mathrm{~nm}$ as nanoparticles, since the distinctive characteristics of these particles may also be observed biologically. [16] Particles with size around 1,000 to 2,000 $\mathrm{nm}$ can be engulfed into cells through macropinocytosis but this may be limited. However the smaller nanoparticles (60 
$\mathrm{nm}$ and below) are more likely to be transported into cells through direct cellular uptake which help to increase bioactivity of encapsulated phenolic phytochemicals. [17]

Two basic approaches are used for creating nanostructures - bottom-up [18] and top-down [19]. The bottom-up technique includes the use of atoms, molecules and nanoparticles as building blocks at a molecular scale. This technique uses chemistry and physics derived technologies that are based on chemical synthesis or strictly controlled mineral growth. The top-down technique engages the breakdown of macroscopic material to a nano-scale through: mechanical (e.g. grinding), chemical (e.g., partial hydrolysis with acids or bases), enzymatic (e.g., treatment with enzymes hydrolyzing cellulose, hemicellulose, pectin and lignin) and physical (e.g. techniques using focused ion beams or high-power lasers). [20]

Surfactants, including polyvinyl alcohol (PVA), are used as stabilizers in many nanoparticle fabrication techniques. PVA is generally white in color and odorless and requires heat $\left(>70^{\circ} \mathrm{C}\right)$ to obtain complete dissolution in water. [21] Surfactants usage regulates the size of the synthesized particles, reduces the dispersion and improves encapsulation effectiveness. PVA is used in many biomedical implementations, for example for wound dressings, cardiovascular devices and drug delivery due to its intoxicity, biocompatibility and excellent mechanical characteristics. [22]

In this work we aimed to evaluate the in-vivo antiinflammatory properties of date seed powder (DSP) nanoparticles and infusion. Specific objectives were; determining the proximate analysis DSP as well as their antioxidant capacity, developing and characterization of nanoparticles of DSP, and finally evaluating the safety and effect of supplementation with DSP on inflammatory markers in rats fed a high fat diet.

\section{Material and Methods}

\subsection{Collection of Material \& Preparation of DSP Sample}

Identification of fruits' families was done by the head of the Fruit department - faculty of agriculture - Alexandria University. Date family is (Barhi).

Date seeds from the Barhi variety were obtained from Azmour Siwa Company. The seeds were soaked in water, washed to get rid of any adhering flesh, air-dried for one day, then roasted in a medium heat oven for one hour and grounded into coarse powder using a hammer mill. Then again ground into fine powder using a commercial home milling machine to produce fine DSP, which was then kept in an air tight container.

\subsection{Proximate Analysis \& Antioxidant Content of DSP}

Total antioxidant capacity, total phenols, total flavonoids were measured by Spectrophotometer UV-VIS Analytikjena Spectro D250 Germany, selenium and zinc were measured by Inductively coupled plasma (ICP MS/MS 8800 Triple Quad) Agilent Technologies in the Agricultural Research Center -
Ministry of Agriculture - Cairo- Egypt. Table 1

\subsection{Preparation of Date Seed Nanoparticles (DSP NP) Using Poly Vinyl Alcohol (PVA Sigma-Aldrich)}

Milling was employed for this purpose as the roasted date seeds were finely ground and infused in distilled water then mixed with PVA aqueous solution. (In order to boost the encapsulation, the surfactant or stabilizer aqueous solution was added to the infused solution then implementing certain stirring requirements including time, speed and temperature of stirring, and centrifugation)

DSP NP1 = Poly vinyl alcohol (PVA) and DSP (1:5 w/w)

$25 \mathrm{~g}$ DSP and $50 \mathrm{~mL}$ distilled water were stirred for $20 \mathrm{~min}$ with a magnetic stirrer at $80^{\circ} \mathrm{C}$ to be mixed at $900 \mathrm{rpm}, 5 \mathrm{~g}$ PVA with $25 \mathrm{ml}$ distilled water were stirred for $20 \mathrm{~min}$ with a magnetic stirrer at $80^{\circ} \mathrm{C}$ to dissolve at $900 \mathrm{rpm}$, and then both aqueous solutions were added together and the final solution was totaled by adding distilled water up to $100 \mathrm{ml}$ and stirred for $1 \mathrm{~h}$ at $80^{\circ} \mathrm{C}$, then; spinning at $4000 \mathrm{rpm} /$ minute for one hour in a centrifuge at room temperature.

DSP NP2 = Poly vinyl alcohol (PVA) and DSP (2:3 w/w)

$15 \mathrm{~g}$ DSP and $50 \mathrm{~mL}$ distilled water were stirred for $20 \mathrm{~min}$ with a magnetic stirrer at $80^{\circ} \mathrm{C}$ to be mixed, $10 \mathrm{~g}$ PVA (i.e., increasing the surfactant or stabilizer concentration) with $25 \mathrm{ml}$ distilled water were stirred for $20 \mathrm{~min}$ with a magnetic stirrer at $80^{\circ} \mathrm{C}$ to dissolve, and then both aqueous solutions added together and the final solution was totaled by adding distilled water up to $100 \mathrm{ml}$ and stirred for $1 \mathrm{~h}$ at $80^{\circ} \mathrm{C}$, then; spinning at $4000 \mathrm{rpm} /$ minute for one hour in a centrifuge at room temperature.

\subsection{DSP Infusion3}

Finally $25 \mathrm{~g}$ DSP and $50 \mathrm{~mL}$ distilled water were stirred for 20 min with a magnetic stirrer at $80^{\circ} \mathrm{C}$ to be mixed, then totaled by adding distilled water to $100 \mathrm{ml}$ and stirred for $1 \mathrm{~h}$ at $80^{\circ} \mathrm{C}$, then; spinning at $4000 \mathrm{rpm} /$ minute for one hour in a centrifuge at room temperature.

\subsection{Characterization}

The products were characterized by using ZETA and IR spectrometer at the Nano-research Unit Faculty of Pharmacy PUA. Scanning electron microscopy was used to verify uniformity of particle shape and size at Faculty of Science, Alexandria, Egypt.

\subsection{Animals and Treatment}

A total of 25 female albino rats (150-180 g) were obtained from the animal house of the faculty of pharmacy and drug manufacturing, Pharos University, Alexandria, Egypt. The rats were acclimated for 3 days before starting the experiment. All animals were housed in standard cages (5 rats/cage), in an air-conditioned rooms at $21-23^{\circ} \mathrm{C}$ and 60 $65 \%$ of relative humidity, and kept on a $12 \mathrm{~h}$ light $/ 12 \mathrm{~h}$ dark cycle. The animals received humane care in accordance with the research ethics for use of animals, issued by the scientific research ethics committee of Pharos University and fed with 
standard laboratory diet and tap water ad libitum.

Ingredients of diet: Standard laboratory diet consists of: $60 \% \mathrm{CHO}, 19 \%$ protein, $6 \%$ fats, $2.3 \%$ fibers and more than $5 \%$ vitamins and minerals. Energy: $3200 \mathrm{Kcal} / \mathrm{kg}$.

Induction of obesity and low grade inflammation by HFD:

High fat diet (HFD) (33\% of calories from fat); $1 \mathrm{~kg}$ (standard laboratory diet), $150 \mathrm{~g}$ plant margarine rich in saturated fatty acids (SFA) from palm oil, and 250g lard.

Experimental groups and protocol

a) Rats were divided randomly into 5 groups comprising 5 rats in each group.

b) Group I: Rats fed on standard laboratory diet for 8 weeks and served as a negative control group.

c) Group II: Rats fed on HFD for 8 weeks; i.e. positive control.

d) Group III: Rats fed on HFD for 4 weeks, and then DSP NP1 by oral gavage $(1 \mathrm{ml} /$ day $)$ in addition to HFD for 4 weeks.

e) Group IV: Rats fed on HFD for 4 weeks, and then DSP NP2 by oral gavage $(1 \mathrm{ml} /$ day) in addition to HFD for 4 weeks.

f) Group V: Rats fed on HFD for 4 weeks, and then DSP Infusion $31 \mathrm{~g} / \mathrm{kg}$ body weight by oral gavage $(1 \mathrm{ml} / \mathrm{day})$ in addition to HFD for 4 weeks.

Measurements of body weight

Body weights of all groups under investigations were measured twice a week

Sample collection: At the end of the experiment, all animals were sacrificed under pentobarbital anesthesia. Blood samples were collected from aorta for biochemical analysis.

Biochemical tests

Samples were tested for C-reactive protein (CRP), Serum amyloid-alpha (SAA), IL-6 and total cholesterol, HDL, total protein, albumin, urea, creatinine, creatine kinase (CK), aspartate aminotransferase (AST), alanine aminotransferase (ALT), alkaline phosphatase (ALP), and gamma-glutamyl transpeptidase (GGT) on Roche/Hitachi Cobas c systems (Integra 400 Plus), Germany by using enzymatic colorimetric methods.

Statistical analysis of the data

Data were fed to the computer and analyzed using IBM SPSS software package version 20.0. (Armonk, NY: IBM
Corp). The Kolmogorov-Smirnov test was used to verify the normality of distribution Quantitative data were described using range (minimum and maximum), mean, standard deviation and median. Significance of the obtained results was judged at the $5 \%$ level.

The used tests were

F-test (ANOVA): For normally distributed quantitative variables, to compare between more than two groups, and

Post Hoc test (Tukey): For pair-wise comparisons

Percentage of change was calculated from the mean of control group according to the following equation:

Percentage of change $=[\mathrm{Mt}-\mathrm{Mc} / \mathrm{Mc}] \times 100-$ Where: Mt, arithmetic mean of treated groups; Mc, arithmetic mean of control group.

The ARRIVE Guidelines Checklist (Animal Research: Reporting In Vivo Experiments) was fulfilled in reporting data of this experiment. [23]

\section{Results}

Proximate analysis and antioxidant content of DSP are shown in table 1.

Table 1. Nutrient content.

\begin{tabular}{ll}
\hline Nutrient content & Date seed powder \\
\hline Carbohydrates & $63.92 \%$ \\
Protein & $7.47 \%$ \\
Fat & $3.01 \%$ \\
Fiber & $19.57 \%$ \\
Ash & $1.78 \%$ \\
Moisture & $4.05 \%$ \\
Total Phenol-gallic acid equivalent & $1319.75 \mathrm{mg} / 100 \mathrm{gm}$ \\
Total antioxidant capacity - ascorbic acid equivalent & $1017.5 \mathrm{mg} / 100 \mathrm{gm}$ \\
Total Flavonoids- quercetin equivalent & $14.75 \mathrm{mg} / 100 \mathrm{gm}$ \\
Zinc & $7.7 \mathrm{mg} / \mathrm{kg}$ \\
\hline
\end{tabular}

Characterization of the products is shown in Table 2 and figures 1,2 and 3.

Table 2. Z-average.

\begin{tabular}{llll}
\hline Item & DSP NP1 & DSP NP2 & DSP Infusion3 \\
\hline ZETA average sizing nm & 371.3 & 397.3 & 3847 \\
ZETA potential & -4.96 & -5.23 & -15.6 \\
Dispersity index & 0.473 & 0.602 & 0.714 \\
\hline
\end{tabular}

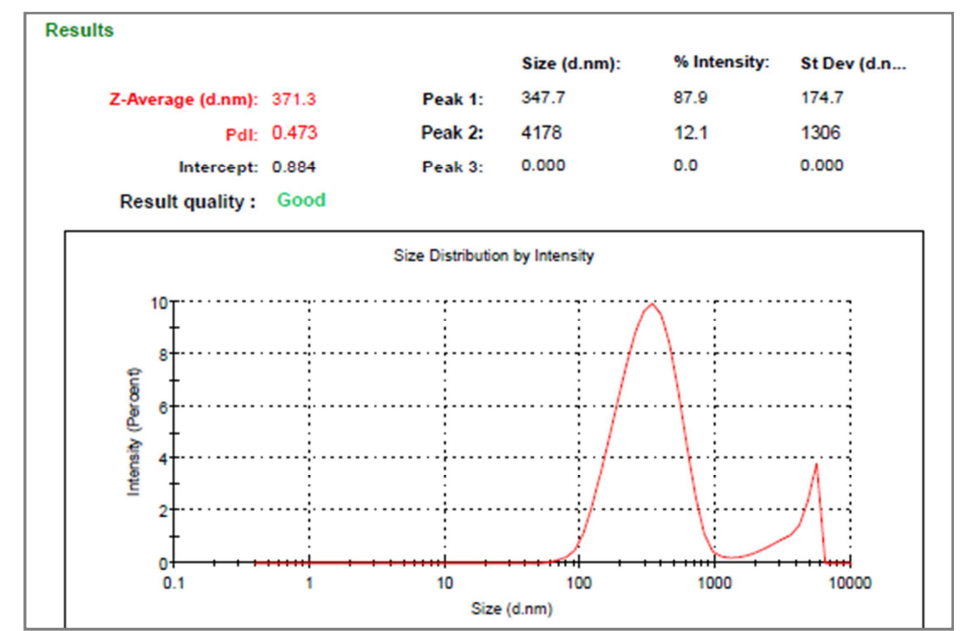



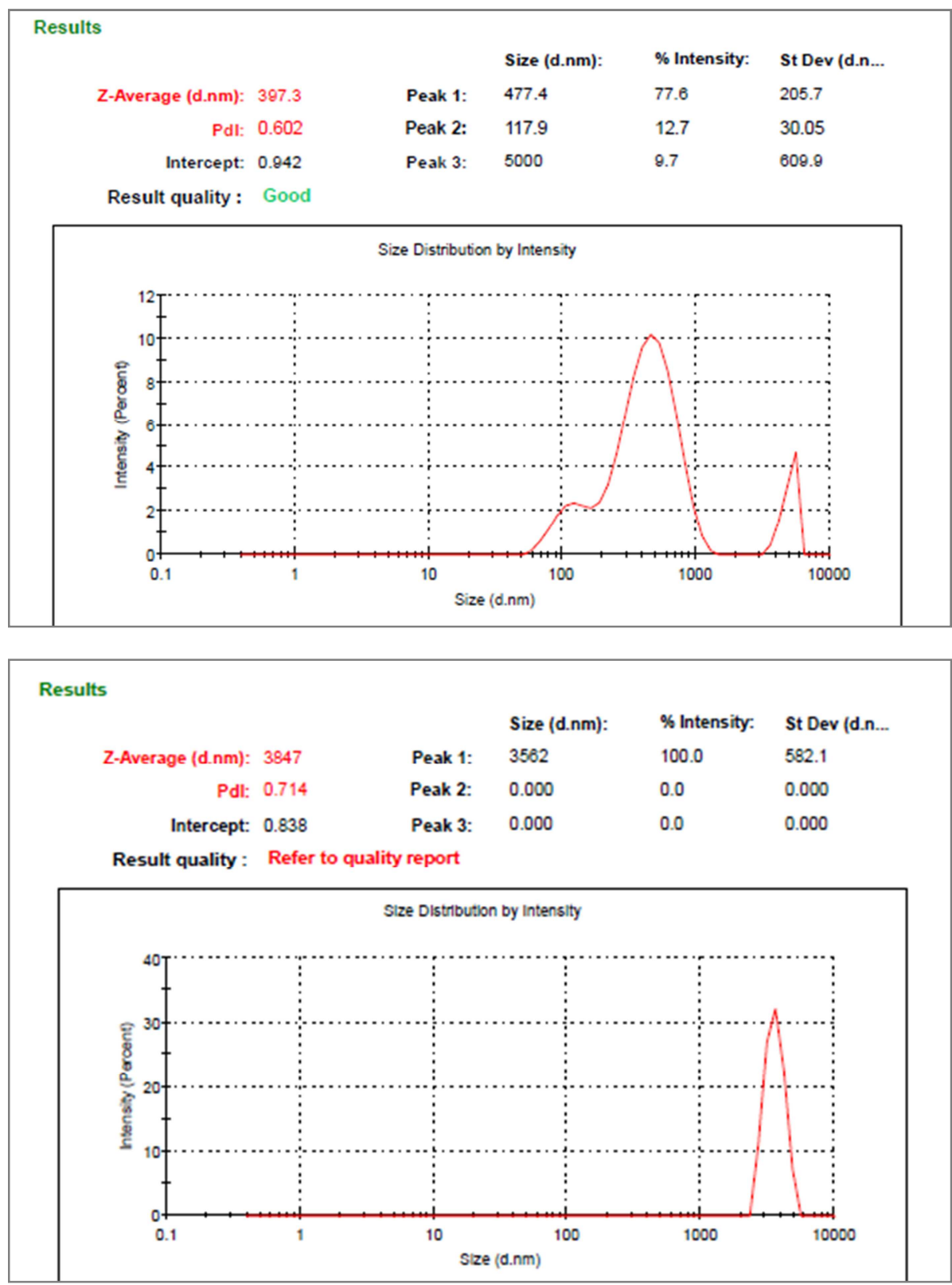

Figure 1. ZITA sizing $(a, b, c)$.

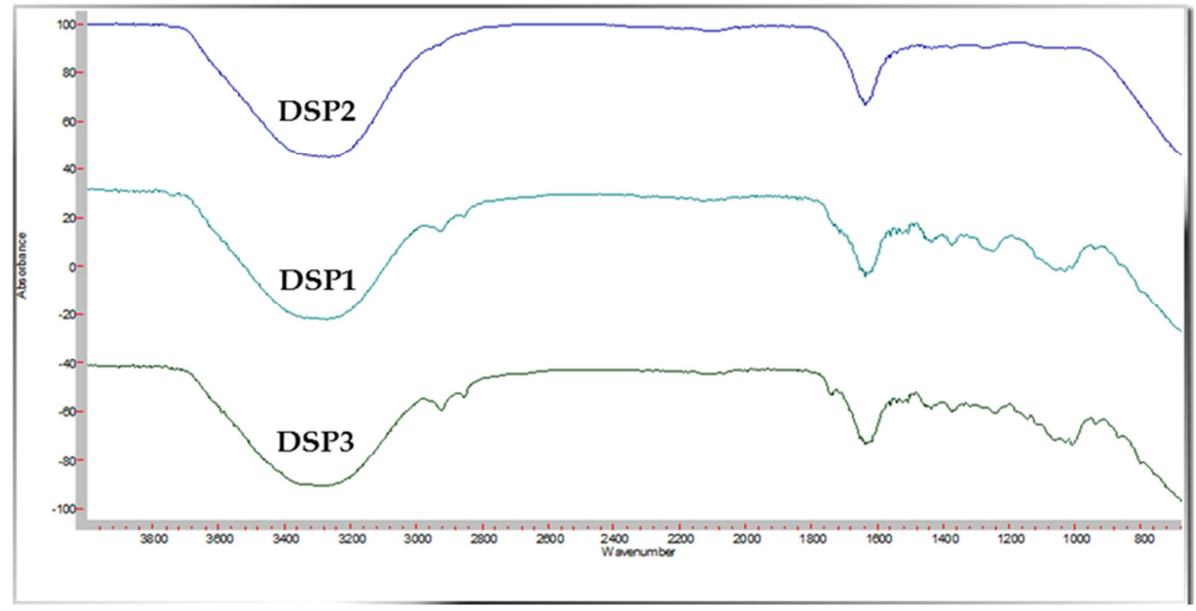

Figure 2. IF-overlay. 


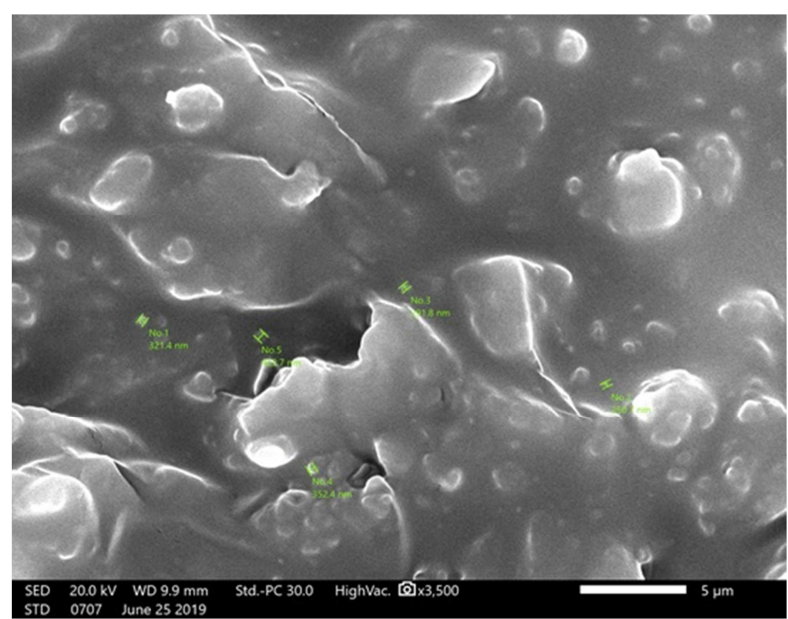

A (5:1)

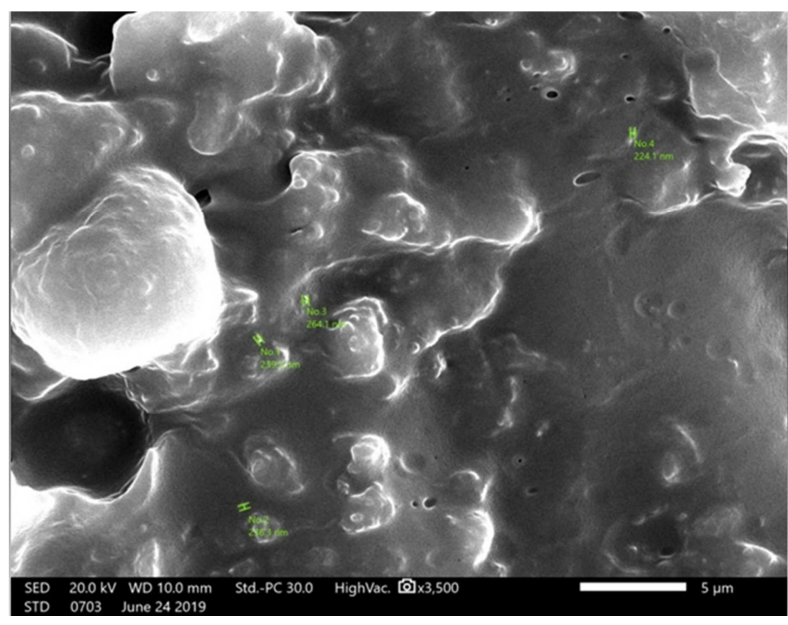

$\mathrm{B}(2: 3)$

Figure 3. SEM images of the prepared DSP NPS.

Table 3. Comparison between the different studied groups according to \% weight change and laboratory investigations.

\begin{tabular}{llllllll}
\hline Parameters & Negative control & Positive control & DSP NP1 & DSP NP2 & DSP Infusion3 & F & p \\
\hline \% weight change & $\uparrow 35.39 \pm 9.74$ & $\uparrow 44.78 \pm 10.61 \mathrm{a}$ & $\uparrow 31.23 \pm 9.40$ & $\uparrow 36.38 \pm 6.67$ & $\uparrow 34.81 \pm 10.93$ & 3.272 & $0.035^{*}$ \\
Creatinine & $0.47 \pm 0.05$ & $0.49 \pm 0.07$ & $0.53 \pm 0.08$ & $0.48 \pm 0.05$ & $0.46 \pm 0.11$ & 0.697 & 0.603 \\
Total cholesterol & $76.2 \pm 14.22$ & $175.6 \pm 10.26 \mathrm{a}$ & $62.0 \pm 13.87 \mathrm{~b}$ & $71.20 \pm 9.73 \mathrm{~b}$ & $61.40 \pm 16.56 \mathrm{~b}$ & $68.114^{*}$ & $<0.001^{*}$ \\
HDL & $62.20 \pm 10.62$ & $63.0 \pm 8.28$ & $53.60 \pm 12.74$ & $53.40 \pm 7.64$ & $53.60 \pm 13.61$ & 1.054 & 0.405 \\
ALT & $44.80 \pm 7.79$ & $47.20 \pm 10.92$ & $50.40 \pm 8.56$ & $41.0 \pm 5.39$ & $50.60 \pm 12.86$ & 0.911 & 0.477 \\
AST & $118.0 \pm 10.12$ & $170.2 \pm 40.43$ & $148.0 \pm 43.07$ & $118.2 \pm 20.54$ & $130.4 \pm 30.54$ & 2.503 & 0.075 \\
Albumin & $4.65 \pm 0.17$ & $4.70 \pm 0.25$ & $4.67 \pm 0.26$ & $4.78 \pm 0.39$ & $4.61 \pm 0.45$ & 0.195 & 0.938 \\
ALP & $39.20 \pm 7.60$ & $67.0 \pm 19.35 \mathrm{a}$ & $43.60 \pm 8.50$ & $46.40 \pm 15.69$ & $51.40 \pm 12.20$ & $3.198^{*}$ & $0.035^{*}$ \\
CRP & $0.84 \pm 0.80$ & $13.40 \pm 4.56 \mathrm{a}$ & $1.04 \pm 0.93 \mathrm{~b}$ & $1.56 \pm 0.74 \mathrm{~b}$ & $1.84 \pm 0.80 \mathrm{~b}$ & $31.222^{*}$ & $<0.001^{*}$ \\
SAA & $6.22 \pm 0.08$ & $11.6 \pm 1.1 \mathrm{a}$ & $7.16 \pm 0.05 \mathrm{~b}$ & $7.34 \pm 0.13 \mathrm{~b}$ & $7.14 \pm 0.05 \mathrm{~b}$ & $90.771^{*}$ & $<0.001^{*}$ \\
IL-6 & $15.54 \pm 0.21$ & $19.92 \pm 5.81$ & $15.50 \pm 0.19$ & $15.66 \pm 0.15$ & $15.72 \pm 0.25$ & 2.753 & 0.057 \\
\hline
\end{tabular}

Means \pm SD are presented. Tukey test was performed to compare rat groups. F: F for ANOVA test. Statistical significance was set at $p \leq 0.05$, a: Statistically significant difference between the Negative control group and other groups; b: Statistically significant difference between the Positive control group and other groups.

Table 3, reveals that the addition of HFD led to significant increase in body weight gain of positive control compared to their corresponding control rats, $\mathrm{P}=0.027$. HFD induced significant elevations in total serum cholesterol and inflammatory markers CRP and SAA levels as compared to the control group; while, HDL, ALT, AST, creatinine and albumin showed no change in all rats.
HFD led to significant increase in total cholesterol in comparison to negative control $(175.6 \pm 10.26)(76.2 \pm 14.22)$ respectively, $\mathrm{p}$ value $(<0.001)$. In all DSP treatment groups, total cholesterol levels were reduced significantly compared to the positive control group. While HDL levels were almost the same in all groups. ALP was significantly higher in positive controls $(67.0 \pm 19.35)$ when compared with negative 
controls (39.20 \pm 7.60$)$, p value (0.028). Yet its level in all DSP treatment groups did not show any significant difference from negative controls.

Other primary biochemical parameters, mentioned in the methods section, done showed no significant difference between all groups. (Not shown in table)

CRP and SAA showed significant elevations in positive control than negative control $p$ value $(<0.001)$, but the increase in IL-6, in spite of being insignificant - $p$ value 0.057 , yet it was notable; $(15.54 \pm 0.21)$ and $(19.92 \pm 5.81)$. In all DSP treatment groups, CRP and SAA showed significant decrease than positive controls.

\section{Discussion}

The identification of highly available sources and remarkable functions is the essence of nutrition research, in a quest for seeking a sustainable and cheap source of nutraceutical compounds we hypothesized that encapsulation of DSP (a plant food waste) in PVA NPs would increase their anti-inflammatory activity in rats fed HFD through increased cellular uptake and we tested their in-vivo bioactivities.

The anti-inflammatory effect of Phoenix dactylifera is related to its unsaturated fatty acids, antioxidant compounds (vitamins E and C) and phenolic compounds. [24] Other findings suggest that dates also play a role in reducing leg swelling and plasma fibrinogen. [25] Date seeds contain greater fat and protein than flesh; as well the seeds are high in fiber and antioxidants and phenolics that add to their antiinflammatory actions. [26-27] In the samples tested, as shown in table 1 , total phenol content of DSP-gallic acid equivalent-was $1319.75 \mathrm{mg} / 100 \mathrm{gm}$, total antioxidant capacity-ascorbic acid equivalent-1017.5 mg/100 gm and total flavonoids content of DSP - quercetin equivalent- was $14.75 \mathrm{mg} / 100 \mathrm{gm}$, while zinc content was $7.7 \mathrm{mg} / \mathrm{kg}$.

\subsection{The Size and Morphology of the DSP NPS}

All work reported on date seed nanoformulation protocols, was for the synthesis of metal NPs (silver and platinum) [28] or by using hydrochloric acid ( $\mathrm{HCl}$ ) [29] or using date seed extract and using chitosan polymer [30] or nanocapsules made from starch polymers [31] and tested mostly for invitro antibacterial activity. In the present study; PVA and distilled water were used to produce date seed nanoparticles.

Badawi JE, et al [30] in 2018, produced nanosized particle ranging from $(152-254 \pm 60 \mathrm{~nm})$; by altering the stirring and centrifugation time, rpm and temperature to reach the optimum conditions which gave their best results using DS extract and chitosan polymer which were; stirring at $500 \mathrm{rpm}$ for $24 \mathrm{hrs}$ and centrifugation at $20000 \mathrm{rpm}$ for $30 \mathrm{~min}$ at room temperature. They showed that their nanoparticles had enhanced antioxidant properties 1.2 folds than the free extract and 100 fold enhanced antibacterial properties than the free extract in vitro. Preparations of NPs of chitosan: Ajwah in ratios is 1:5 and 1:7 were found to be best for sustained effects. NPs prepared by Awad MG. et al [29], had a wide range of size from $1-220 \mathrm{~nm}$; as they filtrated the nanoparticles through a Millipore filter having a pore size of about $220 \mathrm{~nm}$.

Our DSP NPs size and morphology detected by SEM and ZETA ranged from 117-347 $\mathrm{nm}$ as shown in table 2 and figure 1 and 3 . Through increasing the process temperature, the stirring rate, and the quantity of stabilizer, particles were reduced in size. An increase in polyvinyl alcohol concentration caused better nanoparticle qualities. This was consistent with Keum CG, et al. [32]

IR findings published by Bagheri L. et al [31] showed that the IR chart of the free and the loaded preparations did not show significant differences. In contrast; the IR overlay chart in the present study showed some differences between the free and nanosized particle solutions as shown in figure 2.

\subsection{Anti-obesity Effects}

Inflammation is a crucial result of adipose dysregulation in the interpretation of obesity comorbidity. Obesity is therefore a state of fat-induced prolonged inflammation that causes a decrease in immune defense. [33] In the light of HFD consumption and subsequent development of obesity, the increased body weight in positive controls and its amelioration in DSP treated groups (table 3) could be related to the fact that date seed is rich in catechins, as those present in green tea, which have been shown by several studies that they can affect body weight regulation by increasing energy expenditure. [34-35]

\subsection{Safety of DSP NP and Infusion}

There were no significant changes in serum albumin, creatinine as well as, ALT and AST with DSP NPs and infusion as shown in table 3 . Without a change in the primary biochemical parameters following consumption of DSP, the use of DSP could be considered safe for rats. DSP did not substantially alter creatinine which is used in renal function testing. Moreover the rise in ALP -which is an indicator of tissue injury, especially the liver- by HFD; was ameliorated by DSP NPs and infusion, and this is consistent with the results of Al-Rasheed et al. [36] ALP decrease with DSP treatments indicates that DSP did not alter tissue functions and could improve their function through a protective effect on organs. A similar decrease was already observed in a previous study with DSP. [37] Steeped date seed in a dose of $1 \mathrm{~g} / \mathrm{kg}$ was comparable with vitamin $\mathrm{C}$ dose of $1 \mathrm{~g} / \mathrm{kg}$ administration. [38] Saryono $\mathrm{S}$ et al also demonstrated that steeped date seeds can improve GSH peroxidase in premenopausal women. [39]

\subsection{Hypolipidemic Effects}

All DSP groups showed significantly lower total cholesterol levels compared to the positive control group and as HDL levels did not change in all groups; this means that the lowering effect was mainly demonstrated on LDL levels. Findings indicate that DSP NPs and infusion, most probably through their high polyphenol, may help to reduce TC and LDL-C levels in rats as shown in table 3. Diet induced-low 
grade inflammation is one of the underlying mechanisms in the development and enhancement of chronic diseases. In hypercholesterolemic rats DSP may be useful in decreasing symptoms of diseases linked to a low grade inflammatory etiology. Moreover a significant threat to progression of atherosclerosis is obviously an elevated total cholesterol level.

\subsection{The Anti-inflammatory Effect}

Interleukin 6 (IL-6) has pro-and anti-inflammatory characteristics that are context-dependent and is now considered to be a major clinical intervention target. [40] Even though the increase in IL-6 in HFD was not significant, $\mathrm{p}$ value 0.057 , yet it was noteworthy $(15.54 \pm 0.21)$ in negative controls and $(19.92 \pm 5.81)$ in HFD. But till now, the improvement of pro inflammatory cytokines serum levels like IL-6 is not of substantial proof of a real health advantage. [41] Our results did attenuate the elevated levels of interleukin-6 - in spite of being insignificant - with all DSP treatment groups as in table 3 .

Serum Amyloid-A (SAA) is an acute inflammatory sensitive marker. SAA has also been associated with atherosclerosis, like C-reactive protein (CRP). In prospective studies, similar to that for CRP, there has also been a strong independent relationship between SAA and future cardiovascular events. SAA has been independently linked with coronary artery angiographic disease (CAD). Those findings are consistent with the hypothesis that systemic inflammation characterized by high levels of SAA or CRP can promote destabilization of the atherosclerotic plaque and have a possible direct effect on atherogenesis. [42]

Motivated by mounting appeal from clients for wellbalanced nutrition; nanotechnology tools and knowledge are used by researchers to address food and nutrition issues. Recent nano- delivery systems have shown that distinctive phytochemicals improve their oral bioavailability and bioeffectiveness (for example, anti-inflammatory and anticancer). Further attempts to comprehend the possible impacts on the human body and environment of nanoencapsulated phytochemicals are still needed. [43] Both DSP NPs and infusion caused significant decrease in CRP and SAA levels as shown in table 3. Regarding the lack of significance between treatment groups; similar results were obtained by Li et al and others; [44-45] that nanotechnology enabled delivery of plant phytochemicals does not provide an advantage over their free counterpart. Insufficient cellular uptake of the NPs was suggested as a probable reason for the lack of improved efficacy.

Nanosized delivery systems significantly affect the absorption profile of the loaded molecules because the particle size, shape and surface properties of the nanoparticles play a crucial role in the uptake across the gastrointestinal mucosa. The nanocarriers with particle size of $50-300 \mathrm{~nm}$, positive zeta potential and hydrophobic surface were found to have preferential uptake from gastrointestinal tract as compared to their counterparts. [46] Surface charge largely defines nanoparticles interactions with the biological systems. Of note, positively charged nanoparticles show significant cellular uptake compared to negatively charged and neutral nanoparticles. [47]

Thus lack of difference between groups in the current study may be due to the negatively charged NPs and the high dose of infusion used as well as the same used methods for its preparation (i.e., milling, stirring and centrifugation) only without the stabilizer. Comprehensive in-vivo studies are necessary to ascertain improved nutrient bioavailability as one of the most important fields for nanotechnology applications.

This work was mainly intended to examine the anti-obesity effect of DSP and their hypocholesterolemic, antiinflammatory effect as well as safety profile in HFD fed animals. Phytotherapy is regarded as a supplementary approach for prevention and treatment of chronic diseases, although it often does not have adequate scientific evidence based on medical practice. [48] In a study exploring herbals' anti-inflammatory activity; to provide up to date understanding of plants that are precious to researchers in the discipline of natural anti-inflammatory compounds; they argued that synthesized anti-inflammatory drugs have been revealed to be used less frequently recently because they have potential negative hazards and serious adverse reactions. Herbal medicines have become more popular in treating several human disorders in recent decades. Herbs with anti-inflammatory activity are subjects of huge concern, owing to the lack of preparations applicable to human consumption. [49]

Here we provide evidence that DSP is thus a hopeful applicant for reducing inflammatory markers and it could be concluded that DSP can contribute to the prevention of chronic diseases in the light of inflammation as a fundamental etiological factor for these illnesses. Further research is needed to elucidate the underlying mechanisms. Yet; an appealing and a feasible theory for daily use is therefore the consumption of DSP hot infusion intended for raising the anti-inflammatory and antioxidants capacity to fight obesity and its comorbidities in human beings.

\section{Conclusion}

The current study indicates that DSP preparations had antihyperlipidemic and anti-inflammatory activity. They significantly reduce total cholesterol and inflammatory markers levels. We designed DSP NPs and examined their effects in rats fed HFD. We found that both DSP in PVA NPs exhibited the same anti-inflammatory effects compared to their free counterparts. Using PVA NPs of DSP in a wt/wt ratio of 2 PVA: 3 DSP (wt/wt); resulted in an acceptable particle size and physicochemical properties.

\section{Limitations and Future Directions}

More experimentation with various wt/wt ratios and methodologies of DSP NPs is needed to reach more 
acceptable nanoparticles' size and desirable effects. Further studies with larger sample size and longer administration of the ingredients are needed as well. An analysis of and comparison with the date-seed content of polyphenolics and antioxidative contents of prepared NPs should be done to confirm the effectiveness of prepared NPs in further studies.

\section{Competing Interests}

The authors declare that they have no competing interests.

\section{Acknowledgements}

We are grateful for senior nutrition students for their effort in animal data collection.

\section{Authors Contributions}

Amany A Salama and Naglaa M Ismael jointly formulated the major concepts of this paper and provided overall supervision of the experimental part, analyzed the data, revised the final version critically, and approved it and provided information and references for this paper.

Amany A Salama interpreted the lab findings and wrote and edited versions of the paper.

Naglaa M Ismael provided supervision of the animal follow up and data collection.

Mervat M Megeed provided supervision of the nanoparticle fabrication, revised the final version critically and approved it and provided information and references for this paper.

\section{References}

[1] del Castillo MD, Iriondo-DeHond A, Martirosyan DM. Are Functional Foods Essential for Sustainable Health? Ann Nutr Food Sci. 2018; 2 (1): 1015.

[2] Walker C, Gibney ER, Hellweg S. Comparison of environmental impact and nutritional quality among a European sample population - findings from the Food4Me study. Sci Rep. (2018) 8: 2330. doi: 10.1038/s41598-01820391-4.

[3] Varzakas T, Zakynthinos G, Verpoort F. Plant Food Residues as a Source of Nutraceuticals and Functional Foods. Foods. 2016; 5 (4): 88. doi: 10.3390/foods5040088.

[4] Das L, Bhaumik E, Raychaudhuri U, Chakraborty R. Role of nutraceuticals in human health. J Food Sci Technol. 2012 Apr; 49 (2): 173-183.

[5] Kaiser Mahmood, Mohammed S. Alamri, Abdellatif Mohamed Abdellatif, Shahzad Hussain. Date pits (Phoenix dactylifera (L.)) waste to best. Agro Food Industry Hi Tech. 2015; 26 (3): 47-50.

[6] FAOSTAT (2018) Food and Agriculture Organization of the United Nations. http://faostat.fao.org/. Accessed on 20-42019.
[7] Saryono S, Rahmawati E, Hapsari HE, Hidayat AI. Antioxidant enzyme status on rat after date seeds (Phoenix dactylifera) steeping treatment. Int J Res Med Sci. 2016; 4 (6): 1893-1896.

[8] Saryono S et al. Anti-atherogenic properties of Deglet Noor Date seeds (Phoenix dactylifera) Methanol extract on DietInduced Hypercholesterolemic Rats. IOP Conf. Ser: Mater. Sci. Eng. 2017; 172 012046. doi: 10.1088/1757899X/172/1/012046.

[9] Attanzio A, Tesoriere L, Poojary MM, Cilla A. Fruit and Vegetable Derived Waste as a Sustainable Alternative Source of Nutraceutical Compounds. Journal of Food Quality. 2018; 2018: 2 https://doi.org/10.1155/2018/8136190.

[10] Duan, Y., Zeng, L., Zheng, C., Song, B., Li, F., Kong, X., \& $\mathrm{Xu}, \mathrm{K}$. Inflammatory Links between High Fat Diets and Diseases. Frontiers in immunology. 2018; 9: 2649. doi: 10.3389/fimmu.2018.02649.

[11] Forni C, Facchiano F, Bartoli M, et al. Beneficial Role of Phytochemicals on Oxidative Stress and Age-Related Diseases. Biomed Res Int. 2019; 2019: 8748253. doi: 10.1155/2019/8748253.

[12] Min-Hsiung Pan, Ching-Shu Lai and Chi-Tang Ho. Antiinflammatory activity of natural dietary flavonoids. Food Funct. 2010; 1: 15-31 (doi: 10.1039/C0FO00103A).

[13] Hossain KM, Abdal Dayem A, Han J, et al. Molecular Mechanisms of the Anti-Obesity and Anti-Diabetic Properties of Flavonoids. Int J Mol Sci. 2016; 17 (4): 569.

[14] Bilia AR, Isacchi B, Righeschi C, Guccione C, Bergonzi MC. Flavonoids Loaded in Nanocarriers: An Opportunity to Increase Oral Bioavailability and Bioefficacy. Food and Nutrition Sciences. 2014; 5: 1212-1227.

[15] Li, Z., Jiang, H., Xu, C., and Gu, L. A review: Using nanoparticles to enhance absorption and bioavailability of phenolic phytochemicals. Food Hydrocolloids. 2015; 43: 153164.

[16] Roger, E., Lagarce, F., Garcion, E., and Benoit, J. - P. Biopharmaceutical parameters to consider in order to alter the fate of nanocarriers after oral delivery. Nanomedicine. 2010; 5: $287-306$

[17] Ruiz, J. C. R., \& Campos, M. R. S. (Eds.). (2016). New Polymers for Encapsulation of Nutraceutical Compounds. doi: 10.1002/9781119227625. Ch 4. Surface modifications that benefit protein-based nanoparticles as vehicles for oral delivery of phenolic phytochemicals. Zheng Li.

[18] Hatzor-de Picciotto A, Wissner-Gross AD, Lavallee G, Weiss PS. Experimental Nanoscience 2007; 2: 3-11.

[19] Balzani V. Nanoscience and nanotechnology, Pure Appl. Chem. 2008; 80, 8: 1631-1650.

[20] Serkov AT, Radishevskii MB. Nanotechnologies and chemical fibers. Fiber Chemistry 2008; 40: 32-36.

[21] Muppalaneni S, Omidian H. Polyvinyl Alcohol in Medicine and Pharmacy: A Perspective. J Develop Drugs. 2013; 2: 112. doi: 10.4172/2329- 6631.1000112.

[22] Türk CT, Bayindir ZS, Badilli U. Preparation of polymeric nanoparticles using different stabilizing agents. J. Fac. Pharm, Ankara. 2009; 38 (4): 257-268. 
[23] Kilkenny C, Browne WJ, Cuthill IC, Emerson M, Altman DG. Improving Bioscience Research Reporting: The ARRIVE Guidelines for Reporting Animal Research. PLoS Biol. 2010; 8 (6): e1000412.

[24] Rahmani AH, Aly SM, Ali H, Babiker AY, Srikar S, Khan AA. Therapeutic effects of date fruits (Phoenix dactylifera) in the prevention of diseases via modulation of antiinflammatory, anti-oxidant and anti-tumour activity. Int J Clin Exp Med. 2014; 7 (3): 483-91.

[25] Mohamed DA, Al-Okbi SY. In vivo evaluation of antioxidant and anti-inflammatory activity of different extracts of date fruits in adjuvant arthritis. Pol J Food Nutr Sci. 2004; 13 (4): 397-402.

[26] Al-Farsi MA, Lee CY. Nutritional and functional properties of dates: a review. Crit Rev Food Sci Nutr. 2008; 48 (10): $877-$ 87. doi: $10.1080 / 10408390701724264$.

[27] Al-Farsi MA, Alasalvar C, Al-Abid M, Al-Shoaily K, AlAmry M, Al-Rawahy F. Compositional and functional characteristics of dates, syrups, and their by-products. Food Chem. 2007; 104: 943-947.

[28] Haleemkhan AA, Naseem, Vardhini BV. Synthesis of Nanoparticles from Plant Extracts. International Journal of Modern Chemistry and Applied Science. 2015; 2 (3): 195203.

[29] Awad MG., Al Olayan EM., Yehia HM., et al. Method of preparing date palm seed nanoparticles, King Saud University, US9623067B1US Grant, 2017.

[30] Badawi JE, Mamdouh W. Nanoparticle formulation of Phoenix dactylifera L. (Ajwah and Black date) seed and peel and investigating their antioxidant and antibacterial properties [Master's Thesis] The American University in Cairo. 2018. http://dar.aucegypt.edu/bitstream/handle/10526/5600/final\%20 final $\% 20$ for $\% 20$ comittee.pdf?sequence $=3$.

[31] Bagheri L., Madadlou A., Yarmand M., Mousavi M. E. Nano encapsulation of date palm pit extract in whey protein particles generated via desolvation method. Food Res. Int. 2013; 51: 866-871.

[32] Keum CG, Noh YW, Baek JS, et al. Practical preparation procedures for docetaxel-loaded nanoparticles using polylactic acid-co-glycolic acid. Int $\mathrm{J}$ Nanomedicine. 2011; 6: 22252234. doi: $10.2147 /$ IJN. S24547.

[33] Magnuson A, Fouts J, Booth A, Foster M. Obesity-induced chronic low grade inflammation: Gastrointestinal and adipose tissue crosstalk. Integr Obesity Diabetes, 2015 Volume 1 (5): 103-108. doi: 10.15761/IOD.1000124.

[34] Hursel R, Westerterp-Plantenga MS. Catechin- and caffeinerich teas for control of body weight in humans. The American Journal of Clinical Nutrition. 2013; 98: 1682S-1693S.

[35] Lu C, Zhu W, Shen C-L, Gao W. Green Tea Polyphenols Reduce Body Weight in Rats by Modulating Obesity-Related Genes. PLoS ONE. 2012; 7 (6): e38332. https://doi.org/10.1371/journal.pone.0038332.

[36] Al-Rasheed, NM, Attia HA, Mohamad RA, Al-Rasheed NM, Al-Amin MA, AL-Onazi A. Aqueous Date Flesh or Pits Extract Attenuates Liver Fibrosis via Suppression of Hepatic Stellate Cell Activation and Reduction of Inflammatory Cytokines, Transforming Growth Factor- $\beta 1$ and Angiogenic
Markers in Carbon Tetrachloride-Intoxicated Rats. J. Evid. Based Complement. Altern. Med. 2015; 2015: 1-19. https://doi.org/10.1155/2015/247357.

[37] Habib, H. M. and W. H. Ibrahim. Effect of date seeds on oxidative damage and antioxidant status in vivo. J. Sci. Food Agric. 2011; 91 (9): 1674-1679.

[38] Saryono S, Rahmawati E, Hidayat AI, Hisni D, Proverawati A. Plasma Malondialdehyde and Vitamin E Levels after Date Palm Seeds (Phoenix dactylifera) Steeping Administration. Asian Journal of Clinical Nutrition. 2017; 9: 131-6.

[39] Saryono S, Rahmawati E, Proverawati A, Hisni D. Effect of antioxidant status and oxidative stress products in premenopausal women after treatment with date seed powder (Phoenix dactylifera L.): A study on women in Indonesia. Pak J Nutr. 2017; 16 (6): 477-81. doi: 10.3923/ pjn.2017.477.481.

[40] Hunter CA, Jones SA. IL-6 as a keystone cytokine in health and disease. Nature Immunology. 2015; 16 (5): 448-457.

[41] Maggio M, Guralnik JM, Longo DL, Ferrucci L. Interleukin-6 in aging and chronic disease: a magnificent pathway. $J$ Gerontol A Biol Sci Med Sci. 2006; 61 (6): 575-584. doi: 10.1093/gerona/61.6.575.

[42] Johnson BD, Kip KE, Marroquin OS, Ridker PM, Kelsey SF, et al. Serum amyloid A as a predictor of coronary artery disease and cardiovascular outcome in women: the National Heart, Lung, and Blood Institute-Sponsored Women's Ischemia Syndrome Evaluation (WISE). Circulation. 2004; 109: 726-732.

[43] Huang Q, Yu H, Ru Q. Bioavailability and Delivery of Nutraceuticals Using Nanotechnology. JOURNAL OF FOOD SCIENCE. 2010; 75: R50-7.

[44] Li Z, Percival SS, Bonard S, Gu L. Fabrication of nanoparticles using partially purified pomegranate ellagitannins and gelatin and their apoptotic effects. Mol Nutr Food Res. 2011; 55 (7): 1096-1103.

[45] Desai N. Challenges in Development of Nanoparticle-Based Therapeutics. The AAPS Journal. 2012; 14: 282-295.

[46] Roger, E., Lagarce, F., Garcion, E. and Benoit, J.-P. Biopharmaceutical Parameters to Consider in Order to Alter the Fate of Nanocarriers after Oral Delivery. Nanomedicine. 2010; 5: 287-306. http://dx.doi.org/10.2217/nnm.09.110.

[47] Georgieva J. V., Kalicharan D., Couraud P. et al., "Surface characteristics of nanoparticles determine their intracellular fate in and processing by human blood-brain barrier endothelial cells in vitro," Molecular Therapy. 2011; 19: 318325 .

[48] Cravatto G, Boffa L, Genzini L, Garella D. Phytotherapeutics: An evaluation of the potential of 1000 plants. J. Clin. Pharm. Ther. 2010; 35: 11-48.

[49] Beg S, Swain S, Hasan H, Barkat MA, Hussain MS. Systematic review of herbals as potential anti-inflammatory agents: Recent advances, current clinical status and future perspectives. Pharmacogn Rev. 2011; 5 (10): 120-37. doi: 10.4103/0973-7847.91102. 